\title{
Effect of mechanical parameters on dielectric elastomer minimum energy structures
}

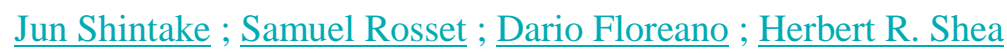

Proc. SPIE 8687, Electroactive Polymer Actuators and Devices (EAPAD) 2013, 86872V (April 9, 2013); doi:10.1117/12.2009368

Copyright 2013 Society of Photo-Optical Instrumentation Engineers. One print or electronic copy may be made for personal use only. Systematic electronic or print reproduction and distribution, duplication of any material in this paper for a fee or for commercial purposes, or modification of the content of the paper are prohibited.

http://dx.doi.org/10.1117/12.2009368 


\title{
Effect of mechanical parameters on dielectric elastomer minimum energy structures
}

\author{
Jun Shintake* $^{\text {a,b }}$, Samuel Rosset ${ }^{\mathrm{a}}$, Dario Floreano ${ }^{\mathrm{b}}$, Herbert R. Shea ${ }^{\mathrm{a}}$ \\ ${ }^{a}$ Microsystems for Space Technologies Laboratory, Ecole Polytechnique Fédérale de \\ Lausanne (EPFL), Neuchâtel, Switzerland \\ ${ }^{\mathrm{b}}$ Laboratory of Intelligent Systems, Ecole Polytechnique Fédérale de \\ Lausanne (EPFL), Lausanne, Switzerland
}

\begin{abstract}
Soft robotics may provide many advantages compared to traditional robotics approaches based on rigid materials, such as intrinsically safe physical human-robot interaction, efficient/stable locomotion, adaptive morphology, etc. The objective of this study is to develop a compliant structural actuator for soft a soft robot using dielectric elastomer minimum energy structures (DEMES). DEMES consist of a pre-stretched dielectric elastomer actuator (DEA) bonded to an initially planar flexible frame, which deforms into an out-of-plane shape which allows for large actuation stroke. Our initial goal is a one-dimensional bending actuator with 90 degree stroke. Along with frame shape, the actuation performance of DEMES depends on mechanical parameters such as thickness of the materials and pre-stretch of the elastomer membrane. We report here the characterization results on the effect of mechanical parameters on the actuator performance. The tested devices use a cm-size flexible-PCB (polyimide, $50 \mu \mathrm{m}$ thickness) as the frame-material. For the DEA, PDMS (approximately $50 \mu \mathrm{m}$ thickness) and carbon black mixed with silicone were used as membrane and electrode, respectively. The actuators were characterized by measuring the tip angle and the blocking force as functions of applied voltage. Different pre-stretch methods (uniaxial, biaxial and their ratio), and frame geometries (rectangular with different width, triangular and circular) were used. In order to compare actuators with different geometries, the same electrode area was used in all the devices. The results showed that the initial tip angle scales inversely with the frame width, the actuation stroke and the blocking force are inversely related (leading to an interesting design trade-off), using anisotropic pre-stretch increased the actuation stroke and the initial bending angle, and the circular frame shape exhibited the highest actuation performance.
\end{abstract}

Keywords: Silicone, PDMS, Dielectric Elastomer, Minimum Energy Structure, DEMES, Bending Actuator, Flexible PCB

\section{INTRODUCTION}

Robots are widely used in industry and in research, and their use is expanding toward other fields such as medical applications. Recently, soft robotics [1-3], an emerging field of robotics, has been attracting much attention. Soft robots, as their name implies, consist mainly of soft or flexible materials in contrast to the rigid parts in conventional robots. This essential attribute, softness, advances robotic functions: adaptive morphology, re-configuration, bio-inspired locomotion, and safe physical human-robot interaction.

There are several soft actuators [1] that can be applied for soft robots such as electroactive polymers, pneumatic actuators and shape memory alloys. Of these, Dielectric Elastomer Actuators (DEA) [4-6] enable large strains (more than $100 \%)$, are lightweight, and can achieve high efficiency [7, 8]. In addition, silicone elastomers such as PDMS (Polydimethylsiloxane) exhibit fast response speed and wide thermal tolerance compared to acrylic elastomers (i.e. 3M VHB) [7-9]. As well as actuation, DEA can act as sensor [10], generator [11] and switch [12], which are promising to construct intelligent integrated soft systems.

Since they are soft, one challenge of DEA is to obtain a configuration transforming strain into useful mechanical motion. For this, many methods have been proposed [13,14]. Dielectric Elastomer Minimum Energy Structures (DEMES) [15], a configuration of DEA proposed by Kofod et al., consist of a pre-stretched DEA bonded to an initially

*E-mail: jun.shintake@epfl.ch 
plane flexible frame. The structure deforms into a configuration that minimizes the mechanical and electromechanical energy, taking a complex shape as the frame bends and the membrane contracts. When a voltage is applied to the DEA, it changes the energy balance between the frame and the membrane, and the frame bending is reduced, till it reverts to its original planar configuration (though often this full actuation range is not attainable due to instabilities or dielectric breakdown at high voltages).

This out-of-plane shape and actuation behavior enable a method for realizing robotic structures and behaviors without hard components. Until now, several different DEMES including modeling have been reported [16-19], and some attempts of developing robots have been presented [20,21]. However, currently there is no result regarding DEMES using PDMS, and, actuation performance such as actuation stroke, output force, and their relation on mechanical parameters to construct actuators have not fully investigated. As mentioned before, PDMS exhibits faster response speed and wide thermal tolerance compared to VHB, which enhance robotic systems in terms of controllability of the motion and survivability in the external environment.

The objective of this study is to develop a compliant structural actuator for a soft robot which will use interconnected, self-sensing, PDMS-based DEMES. Currently we aim at a one-dimensional bending actuation with 90 degree stroke in size of $\mathrm{cm}$-scale. In this paper, we characterize DEMES focusing on mechanical parameters to design the actuators. During the experiments, actuation stroke and blocking force are measured, and the mechanical energy done by force is calculated. Section 2 describes the detail of the mechanical parameters that were taken into account for the characterization. Section 3 shows the fabrication process of DEMES used in this work. The actuators were then measured, and their actuation stroke and blocking force are reported in section 4 . Section 5 discusses experimental results. Section 6 summarizes the output of this research and future work.

\section{MECHANICAL PARAMETERS OF DEMES}

Two main factors can be considered as the actuation performance of DEMES: 1 . the actuation stroke between the initial shape at no applied voltage and the shape at maximum applied voltage, and 2 . the force generated by the deformation. The initial shape corresponds to minimization of the sum of bending energy of the frame and of the strain energy of the DEA. The desired initial shape can be controlled by adjusting mechanical parameters of the DEA such as pre-stretch ratio, planar shape and area, thickness, and elastic modulus. The actuation stroke changes with release of the bending energy of the frame corresponding to actuation strain of the DEA. Therefore, if the bending stiffness of the frame is changed, the stroke is smaller at higher stiffness, and larger at lower stiffness. Similarly, the output force is larger at higher stiffness, and smaller at lower stiffness. The bending stiffness changes with mechanical parameter such as, planar shape and its area, thickness, and elastic modulus. To improve the actuation performance, optimization of prestretch condition can be considered. Recent results show that anisotropic bi-axial pre-stretch can increase the actuation stroke [18]. Since the pre-stretch ratio changes the initial strain energy of the DEA, it is necessary to adjust the parameters of the frame and the DEA corresponding to the pre-stretch. The parameters mentioned above are not independent, but related each other. This relation can affect the actuator performance, often with complex shape, and make the designing for prototyping and optimization difficult. In order to understand the effects of the mechanical parameters and their relation, and gain designing criteria, we characterized the actuators by changing one parameter at a time.

The mechanical parameters considered for the characterization are summarized in Table 1. Figure 1 is a schematic of the parameters. In the figure, we considered the width of the actuators in the $x$ direction, length in the $y$ direction, and thickness in the $z$ direction. The frame geometry is represented by its width $W_{f}$, length $L_{f}$, and shape in the $x$ - $y$ plane. Similarly, the DEA geometry is represented by width $W_{D}$, length $L_{D}$, and shape in the $x-y$ plane. In Table 1 , pre-stretch ratio $(X: Y)$ means combination of pre-stretches on the width direction $(X)$ and on the length direction $(Y)$. The bending stiffness is affected by the geometrical parameters of both the frame and the DEA. Since the elastic modulus of the DEA is much smaller than that of the frame, the bending stiffness of the DEA is ignored. For characterization, three sets of actuators were prepared. One parameter was changed for each of the sets, in order to see its effect on the actuator performance: set (a) had different frame width $W_{f}(16,21$, and 30mm), set (b) had different pre-stretch ratio $(X: Y)(1: 1.3$, 1.3:1.3, 1.95:1.3, and 1.3:1.95), and set (c) had different frame shapes (rectangular, circular, and triangular). Regardless of the frame shape and size, the electrodes of DEA covered the entire suspended membrane. Additionally, the surface of the suspended membrane was kept identical for all the different samples. The tip angle and blocking force of the actuators were then measured as functions of the applied voltage. The actuation stroke, and the mechanical energy were calculated from the measured data. 

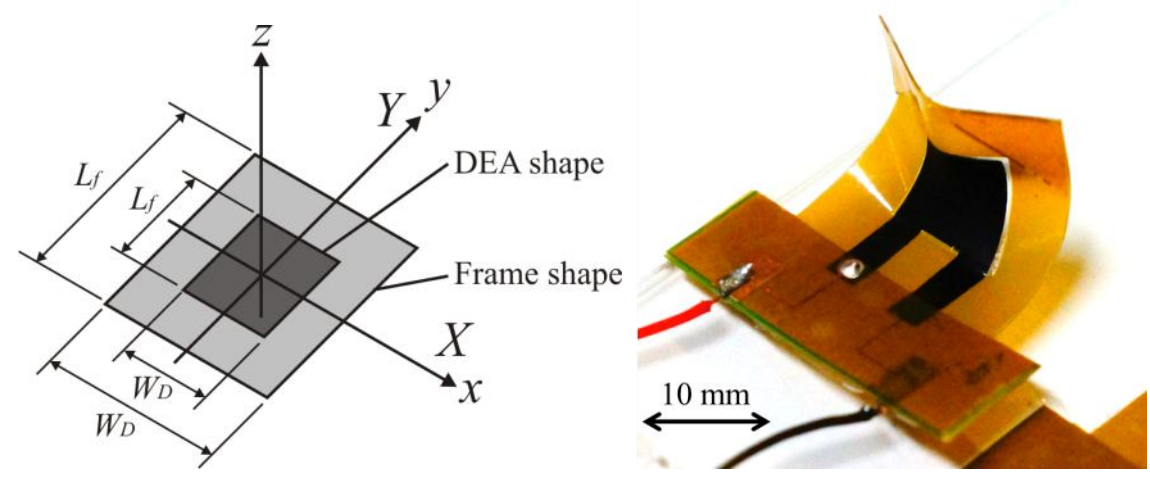

Figure 1. (Left) schematic of the mechanical parameters, and (right) a rectangular DEMES actuator fabricated in this study (electrode is black covering the entire silicone membrane).

Table 1. Mechanical parameters of DEMES and condition for the experiments.

\begin{tabular}{|c|c|c|c|c|}
\hline \multicolumn{2}{|c|}{ Condition for the experiments } & Different frame width & $\begin{array}{c}\text { Different pre-stretch } \\
\text { condition }\end{array}$ & Different frame shape \\
\hline \multirow{5}{*}{ Frame geometry } & Thickness & $50 \mu \mathrm{m}$ & $50 \mu \mathrm{m}$ & $50 \mu \mathrm{m}$ \\
\hline & Width $W_{f}$ & 16,21 , and $30 \mathrm{~mm}$ & $21 \mathrm{~mm}$ & NA \\
\hline & Length $L_{f}$ & 21 & $21 \mathrm{~mm}$ & \begin{tabular}{|c|} 
NA \\
\end{tabular} \\
\hline & Shape & Rectangular & Rectangular & $\begin{array}{c}\text { Rectangular, circular, } \\
\text { and triangular }\end{array}$ \\
\hline & Area of shape & $320 \mathrm{~mm}^{2}$ & $320 \mathrm{~mm}^{2}$ & $320 \mathrm{~mm}^{2}$ \\
\hline \multirow{6}{*}{ DEA geometry } & $\begin{array}{c}\text { Thickness before } \\
\text { pre-stretch }\end{array}$ & $50 \pm 5 \mu \mathrm{m}$ & $50 \pm 5 \mu \mathrm{m}$ & $50 \pm 5 \mu \mathrm{m}$ \\
\hline & Width $W_{D}$ & $10 \mathrm{~mm}$ & $10 \mathrm{~mm}$ & NA \\
\hline & Length $L_{D}$ & $10 \mathrm{~mm}$ & $10 \mathrm{~mm}$ & NA \\
\hline & Shape & Rectangular & Rectangular & $\begin{array}{l}\text { Rectangular, circular, } \\
\text { and triangular }\end{array}$ \\
\hline & Area of shape & $100 \mathrm{~mm}^{2}$ & $100 \mathrm{~mm}^{2}$ & $100 \mathrm{~mm}^{2}$ \\
\hline & Pre-stretch ratio & $1: 1.3$ & \begin{tabular}{|c|}
$1: 1.3,1.3: 1.3$, \\
$1.95: 1.3$, and $1.3: 1.95$
\end{tabular} & $1.95: 1.3$ \\
\hline \multirow{2}{*}{ Modulus of elasticity } & Frame & $4.8 \mathrm{GPa}[22]$ & $4.8 \mathrm{GPa}$ & $4.8 \mathrm{GPa}$ \\
\hline & DEA* & $\sim 1 \mathrm{MPa}[23]$ & $\sim 1 \mathrm{MPa}$ & $\sim 1 \mathrm{MPa}$ \\
\hline
\end{tabular}

*The modulus of elasticity of the DEA depends on curing temperature

The characterization was aimed at understanding the influence of the following parameters.

a) Frame width

Three different frame widths were considered in order to change the bending stiffness of the frame and to expose the trade-off between the actuation stroke and the output force.

b) Pre-stretch ratio

Four different pre-stretch combinations were tested. Uniaxial pre-stretch is the most intuitive approach, but recent results show that anisotropic bi-axial pre-stretch can increase the actuation stroke [18].

c) Frame geometry

Three different frame shapes were tested in order to study its impact on the actuation performance such as on the stroke, the force, and their trade-off. 


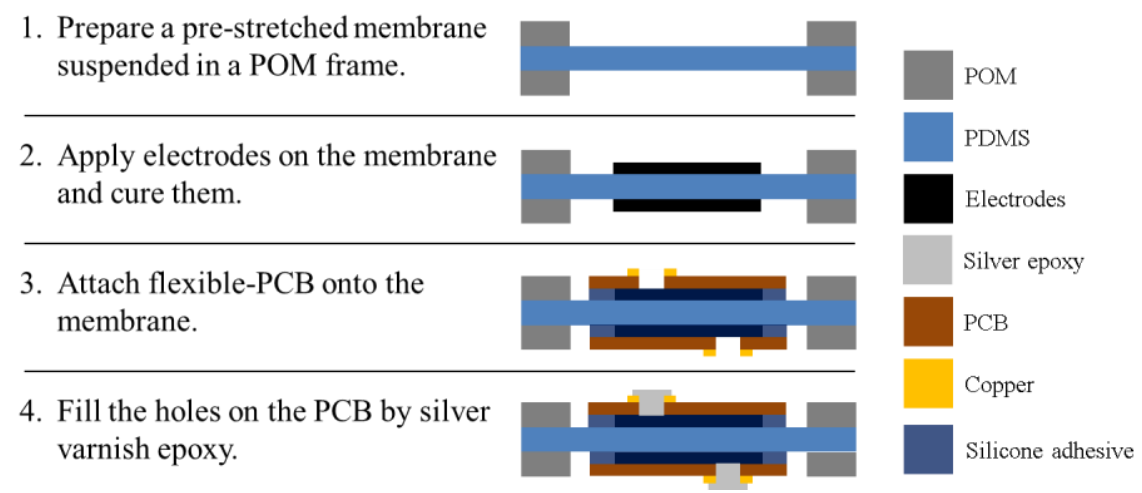

Figure 2. Fabrication process of the DEMES actuators.

\section{ACTUATOR FABRICATION}

The actuators were fabricated following the process shown in Figure 2. A PDMS (Dow-Corning Sylgard 186) membrane was casted with a film coater (Zehntner ZAA2300) and a film applicator (Zehntner ZUA2000), and crosslinked for 3 hours at $60{ }^{\circ} \mathrm{C}$, which lead to membrane of about $50 \mu \mathrm{m}$ thickness. The membrane was then pre-stretched corresponding to the experimental conditions shown in Section 4, and suspended on a support made of POM (Polyoxymethylene). The electrodes consist of a soft silicone mixed with carbon black and were applied on the pre-stretched membrane using a pad-printing machine (Teca-Print TPM-101), and then cured for 1 hour at $60{ }^{\circ} \mathrm{C}$. The frame was then glued on the membrane using a silicone adhesive (Nusil R32-2186), which was applied on the membrane by pad-printing. The frame consists of a polyimide flexible-PCB (Dupont AP9121R) of $50 \mu \mathrm{m}$ thickness. The frame was cut by either a plotter cutter (RoboPro CE5000-40-CRP) or a laser cutting system (Trotec Speedy300). The whole device was put in the oven for 1 hour at $60{ }^{\circ} \mathrm{C}$ to cure the adhesive. After the curing, the holes on the PCB were filled with a conductive silver vanish in order to provide electrical connection with the electrodes. Finally, the actuator was released from the support by cutting the dielectric membrane using a scalpel.

Unlike simple plastic frame, use of flexible-PCB enhances connection between the DEA and the frame to be able to provide electrical signal from controller, and eases construction of systems where several actuators are connected together including other electrical parts. The flex-PCB allows for much smarter systems, with easy signal routing, with the PCB serving both mechanical and electrical purposes. 

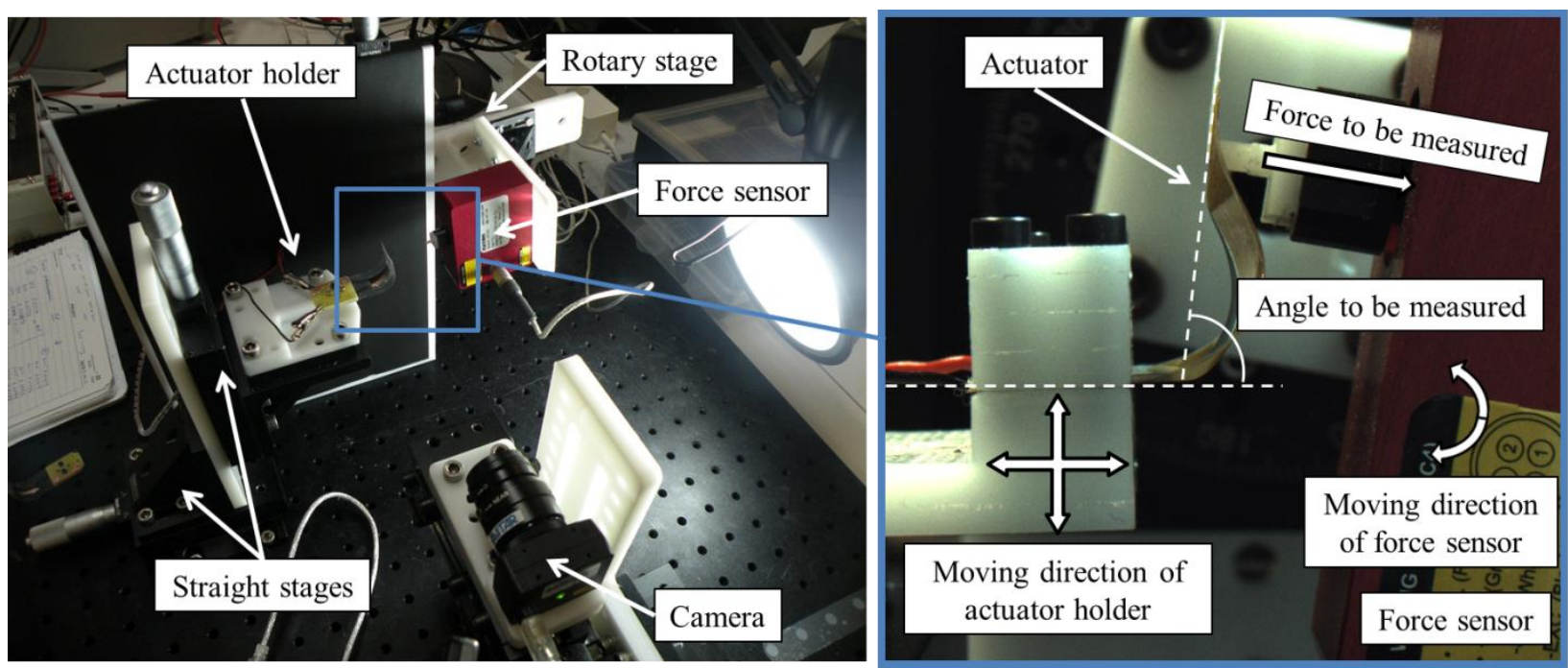

Figure 3. (Left) measurement system, and (right) methods for measuring the tip angle and the blocking force.
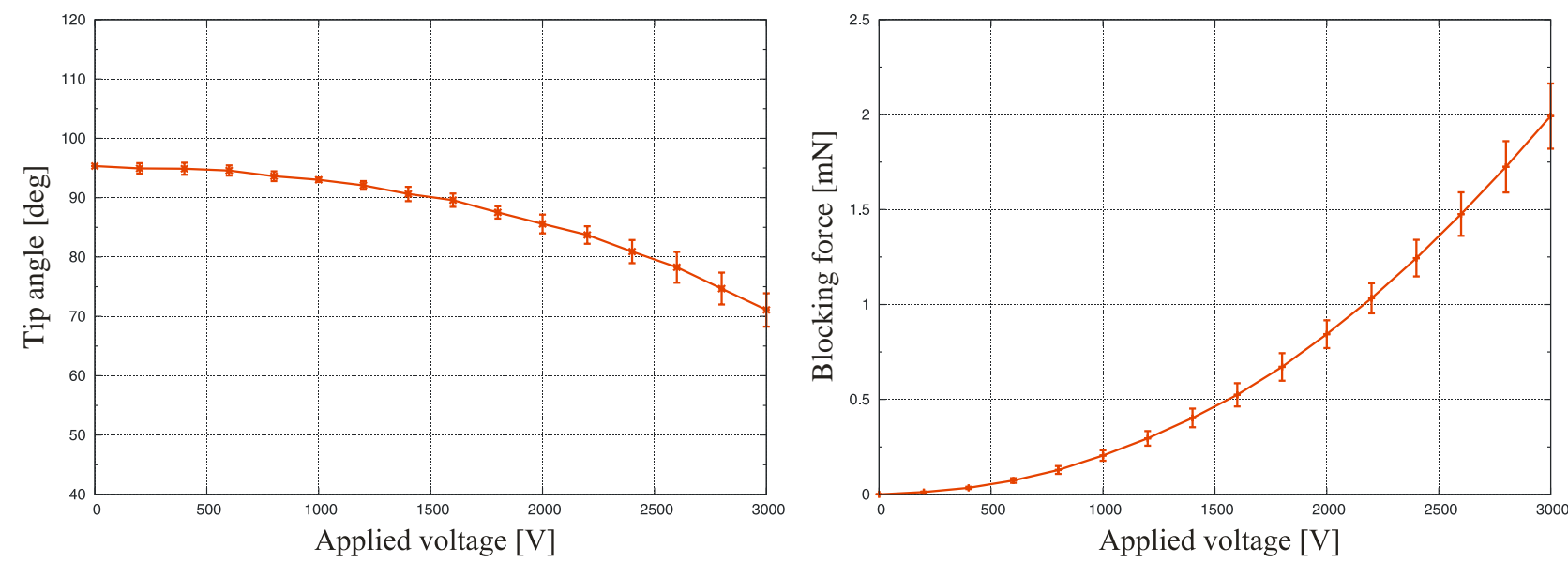

Figure 4. (Left) tip angle and (right) blocking force of earlier generation actuators made with ion-implanted electrodes, both plots are the average of 7 actuators. The tip angle decreases as a function of voltage from its initial angle, while the blocking force increases.

\section{EXPERIMENTAL RESULTS}

\subsection{Experimental setup}

The measurements were conducted on the system shown as Figure 3. To characterize the actuation stroke, we measured the tip angle of the actuators observed from lateral side, shown on the right side of Figure 3. For this, a CMOS camera (Point Grey FMVU-13S2C) was used in combination with a Matlab script in order to extract either curvature of the frame or the angle of the tip from horizontal line. A load cell (FUTEK LRF400) was used to measure blocking force perpendicular to the actuator tip. The position of the actuator holder, the camera and the force sensor were adjusted by precision stages. Figure 4 shows the previously measured result of seven actuators made with ion-implanted electrodes [24], showing tip angle and blocking force as functions of the applied voltage. A large part of the error in measurement of the angle came from fabrication variations, which meant that the initial angle at $0 \mathrm{~V}$ varied in different cases. On the actuators shown in Figure 4, average initial angle of $95.3 \pm 4.8$ deg was measured. 


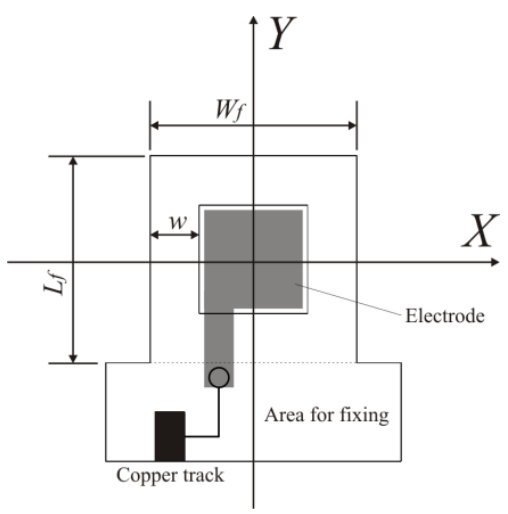

Table 2. Summary of measured tip angle and actuation stroke on different frame width.

\begin{tabular}{|c|c|c|c|}
\hline Beam width $w(\mathrm{~mm})$ & 2.5 & 5 & 10 \\
\hline Tip angle at 0 V $(\mathrm{deg})$ & 113.4 & 81.1 & 51.4 \\
\hline Tip angle at 3 kV $(\mathrm{deg})$ & 93.1 & 70.0 & 46.0 \\
\hline Actuation stroke $(\mathrm{deg})$ & 20.3 & 11.1 & 5.4 \\
\hline Blocking force at $3 \mathrm{kV}(\mathrm{mN})$ & 3.0 & 4.3 & 4.7 \\
\hline
\end{tabular}

Figure 5. Schematic of the rectangular frame.
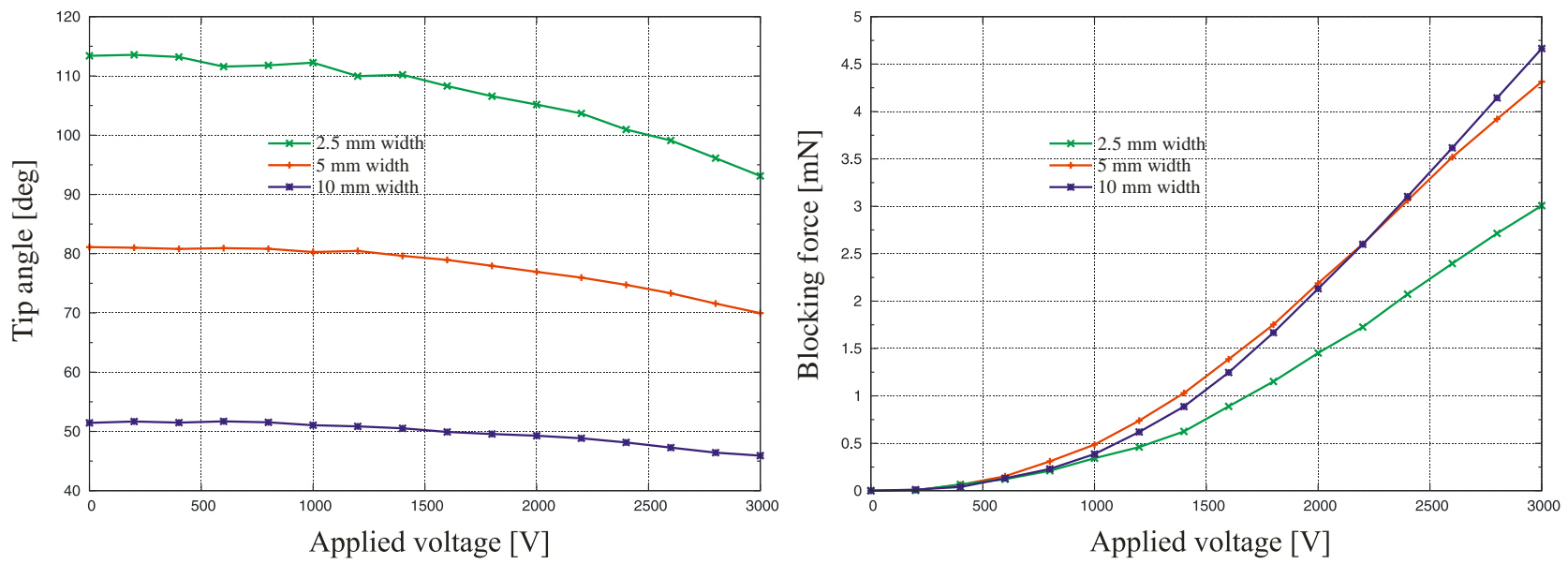

Figure 6. Results of the measurement on different frame width: (left) tip angle versus applied voltage, and (right) blocking force versus applied voltage. The tip angle is shifted corresponding to the frame width, and results in different stroke angle. On the other hand, maximum force is also shifted with the frame width.

\subsection{Experiment on different frame width}

The measured actuators had rectangular shape shown in Figure 5, and specification shown in Table 1. The electrode shape of the actuators follows the outline of the frame. In the table, the pre-stretch ratio used for the DEA represents combination of pre-stretch ratios applied on: width direction as $X$, and length direction $Y$. Width $w$ is the distance between the frame outline and the electrode outline. We tested three different frame widths on the actuators. Four actuators were measured: one for $w=2.5 \mathrm{~mm}$, two for $w=5 \mathrm{~mm}$, and one for $w=10 \mathrm{~mm}$. For the measured values of actuators which have $5 \mathrm{~mm}$ beam width, average was taken. Figure 6 (left) shows measured tip angle as a function of applied voltage. Tip angles are shifted by around $30 \mathrm{deg}$ at initial state $(\mathrm{V}=0 \mathrm{~V})$, and decreasing with increasing applied voltage. Table 2 shows the summary of tip angle and actuation stroke obtained from the experiment. The initial tip angle and the actuation stoke appears to change proportionally with width $w$.

Figure 6 (right) shows the blocking force as a function of applied voltage. The force increases with the voltage. At applied voltage of $3 \mathrm{kV}$, the actuator of $10 \mathrm{~mm}$ width shows largest force while $2.5 \mathrm{~mm}$ shows smallest value. The maximum force of each actuator is summarized in Table 2. 

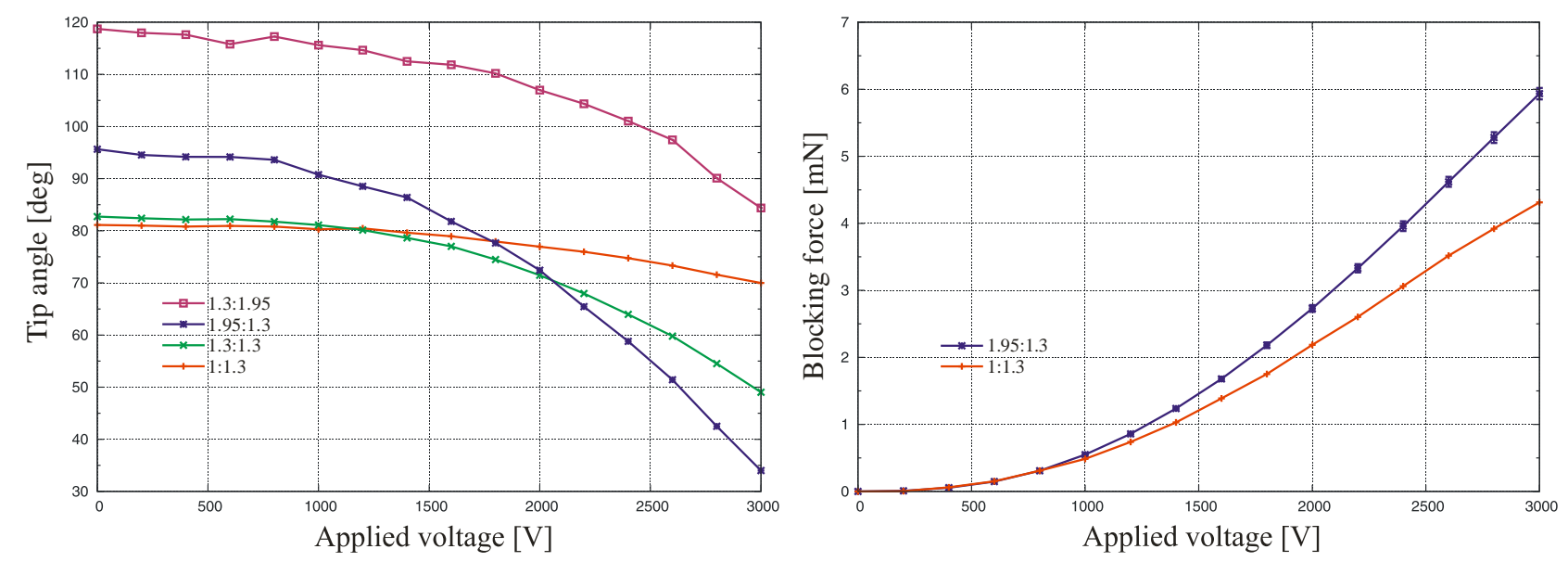

Figure 7. Results of the measurement on different frame shape: (left) tip angle versus applied voltage, and (right) blocking force versus applied voltage with 1:1.3 and 1.95:1.3 pre-stretch ratios. The actuators are showing different initial angle with their pre-stretch ratio on $Y$ direction. The actuator with 1.95:1.3 shows largest actuation stroke, and has larger blocking force.

Table 3. Summary of measured tip angle and actuation stroke on different pre-stretch condition.

\begin{tabular}{|c|c|c|c|c|}
\hline Pre-stretch ratio $(X: Y)$ & $1: 1.3$ & $1.3: 1.3$ & $1.95: 1.3$ & $1.3: 1.95$ \\
\hline Tip angle at $0 \mathrm{~V}(\mathrm{deg})$ & 81.1 & 82.7 & 49.0 & 33.7 \\
\hline Tip angle at $3 \mathrm{kV}(\mathrm{deg})$ & 70.0 & 95.7 & 34.0 & 61.7 \\
\hline Actuation stroke $(\mathrm{deg})$ & 11.1 & 118.7 & 84.4 & 34.3 \\
\hline Blocking force at $3 \mathrm{kV}(\mathrm{mN})$ & 4.3 & - & 5.9 & - \\
\hline
\end{tabular}

\subsection{Experiment on different pre-stretch condition}

Four different combinations of pre-stretch ratios were used for the actuators: 1:1.3, 1.3:1.3, 1.95:1.3, and 1.3:1.95 in the directions refer to $X: Y$ shown in Figure 5. All the actuators had rectangular frame shape same as the one used in subsection 4.2. The number of measured actuators was 3 for each pre-stretch condition, and then average was taken. Figure 7 (left) shows the result of measuring tip angle as a function of applied voltage. In the figure, initial angles are different among the conditions. Table 3 summarizes initial tip angle, variation of tip angle, and actuation stoke on each condition. Actuation stroke is largest on 1.95:1.3 while 1:1.3 shows smallest value. Figure 7 (right) shows the blocking force as a function of applied voltage on one actuator with pre-stretch condition of 1.95:1.3 and 1:1.3. The data of 1.95:1.3 includes standard deviation on every point taken from five measurements. The actuator made with 1.95:1.3 shows larger force compared to 1:1.3. The maximum force of each actuator is summarized in Table 3 .

\subsection{Experiment on different frame geometry}

Three different frame shapes, as shown in Figure 8, were used on the actuators: rectangular, circular, and triangular. Specification of the actuators is shown in Table 1 and Table 4. The frame shapes were designed to have same area. For the pre-stretch ratio, 1.95:1.3 was used, because this combination produced best performance in previous experiment. Figure 9 (left) shows the measured tip angle as a function of applied voltage. In the data, circular shape shows largest stroke while the stroke of triangular shape is smallest. The result of measured blocking force at initial tip angle is shown in Figure 9 (right). Similar to tip angle result, circular shape shows largest force while triangular shape is smallest. The actuators were then characterized by their mechanical energy. For this, the blocking force and the length of movement corresponding to the tip angle at every $100 \mathrm{~V}$ step were measured. Figure 10 shows the method we used to measure the blocking force with the tip angle. The probe of the force sensor was put on the tip corresponding to applied voltage $(<3$ $\mathrm{kV}$ ), and then the voltage was increased to $3 \mathrm{kV}$ for measuring maximum force. The measured force as a function of tip angle is shown in Figure 12 (left). In the figure, circular shape shows larger force at every angle. The mechanical energy was then calculated from the data. For the calculation, following equations were used. 

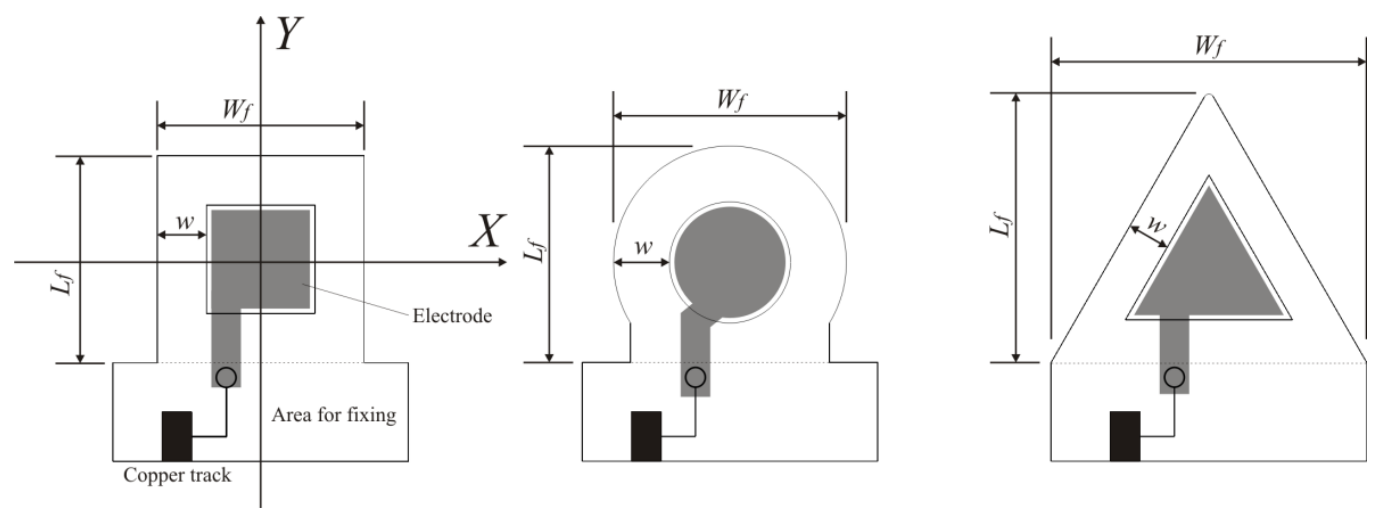

Figure 8. Schematic of rectangular, circular, and triangular shape.

Table 4. Specification of the actuators with different frame shape.

\begin{tabular}{|c|c|c|c|}
\hline Frame shape & Rectangular & Circular & Triangular \\
\hline Frame width $W_{f}(\mathrm{~mm})$ & 21.0 & 23.6 & 32.0 \\
\hline Frame length $L_{f}(\mathrm{~mm})$ & 21.0 & 22.0 & 27.7 \\
\hline Width $w(\mathrm{~mm})$ & 5.0 & 5.7 & 4.4 \\
\hline
\end{tabular}
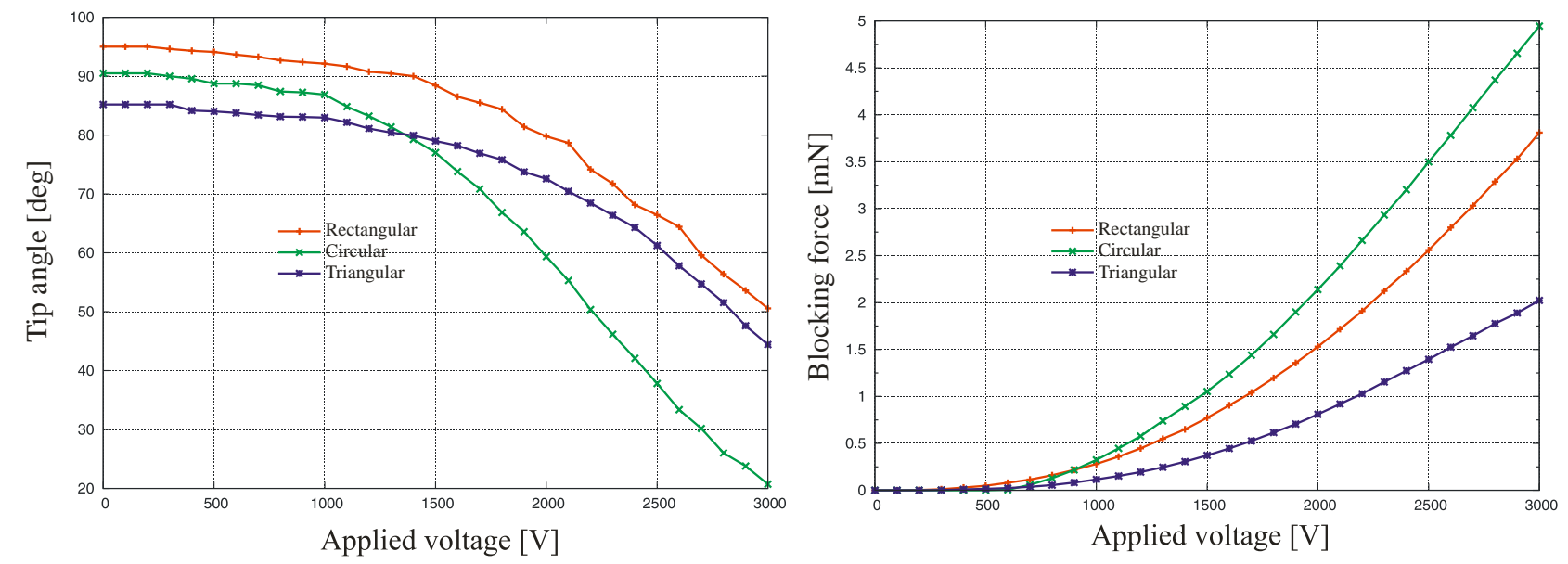

Figure 9. Results of the measurement on different frame shape: (left) tip angle versus applied voltage, and (right) blocking force versus applied voltage. Circular shape shows largest actuation stroke. The actuators show clear difference of blocking force, and the force is maximum on circular shape.

$$
\begin{gathered}
U_{m}=\sum_{i=0}^{N} F_{i}(V) \cdot \Delta l \\
\Delta l=P_{i+1}(y, z)-P_{i}(y, z)
\end{gathered}
$$

where $F_{i}$ is the blocking force at the tip angle as a function of the voltage $V$ applied with every $100 \mathrm{~V}$ step, and $\Delta l$ is the length of the movement of the tip calculated with the tip position $P_{i}$. The number of measurement points $N$ was $31(100 \mathrm{~V}$ step in the range of $0-3 \mathrm{kV}$ ). In equation (1), the mechanical energy $U_{m}$ is represented by the work performed by the blocking force and the length of the movement of the tip. $\Delta l$ was calculated by measuring distance difference of the tip position where the probe was put. The tip position was defined with length direction $y$, and vertical direction $z$, as shown in Figure 11. The actuation stroke $(\mathrm{mm})$ was then extracted as sum of $\Delta l$ at the applied voltage range of $0-3 \mathrm{kV}$. Figure 13 (left) plots actuation stroke on the plane $(y, z)$. Since all the shapes have different frame length $L_{f}$, tip position on the vertical direction is higher at triangular shape. Circular shape shows longest trajectory as it has largest stroke angle. 


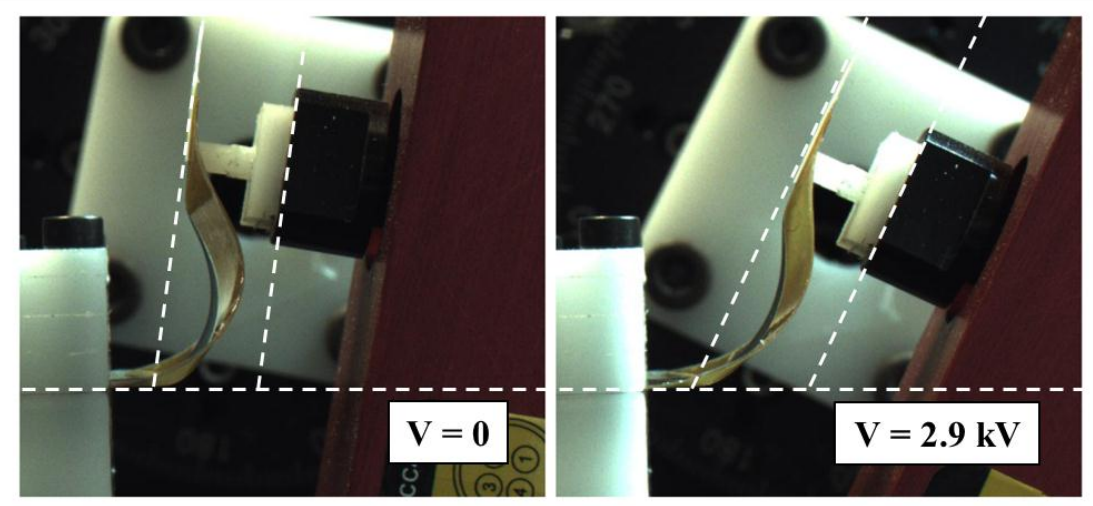

Figure 10. Schematic of the force measurement with different tip angle. The probe of the force sensor was put perpendicular to the actuation tip, at every $100 \mathrm{~V}$ step.
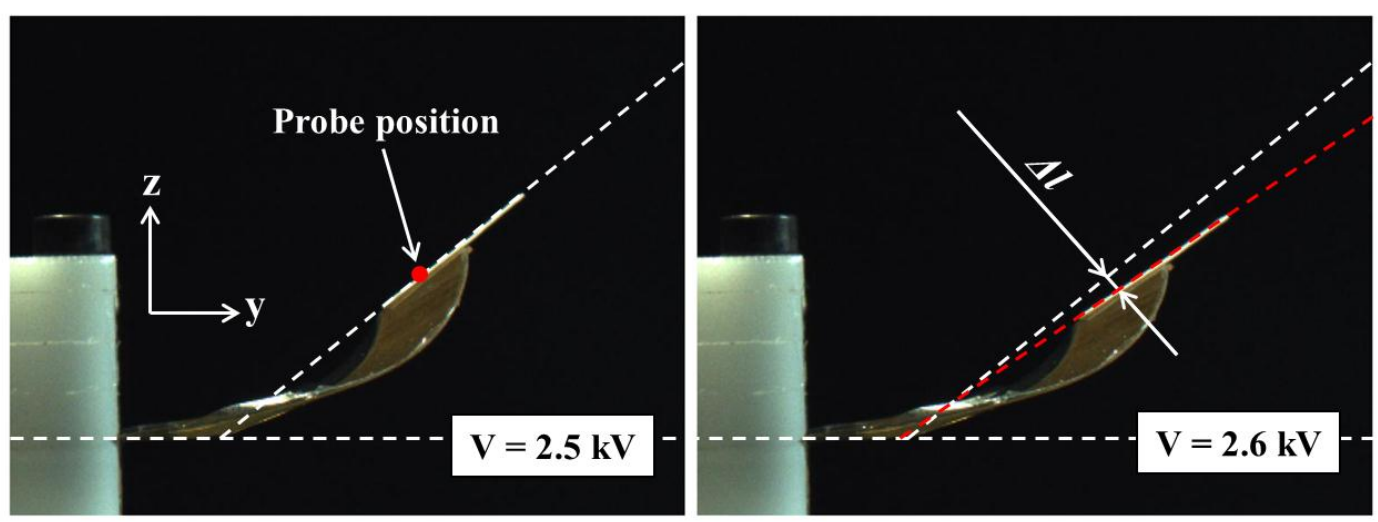

Figure 11. Schematic of the length of the measurement for calculating actuation stroke. The length was measured for every $100 \mathrm{~V}$ step.

Figure 12 (right) shows the actuation stroke versus blocking force. In this figure, the area bounded by the axes and the curve represents the mechanical energy. The energy was highest at circular shape, and lowest at triangular shape. The electromechanical efficiency of the actuators was also calculated. Electrical energy of the DEA, $U_{\mathrm{DEA}}$ can be written as:

$$
\begin{gathered}
U_{D E A}=\frac{1}{2} C V^{2} \\
C=\varepsilon_{0} \varepsilon_{r} \frac{A}{d}
\end{gathered}
$$

where $C$ is the capacitance, $\varepsilon_{0}$ is the permittivity of the free space, $\varepsilon_{r}$ is the relative permittivity of the elastomer, $A$ is the area of the electrode, and $d$ is the thickness of the elastomer at initially pre-stretched state. Strictly, it is necessary to use the actual value of $d$ and $A$ in the actuator which is deforming, however it is difficult to measure. Therefore we consider that equation (3) which gives an approximation of the electrical energy of the DEA. With the mechanical energy, the electromechanical efficiency $\eta$ can be derived as follows.

$$
\eta=\frac{U_{m}}{U_{D E A}}
$$

The maximum electromechanical efficiency was $13.6 \%$ at circular shape. Figure 13 (right) shows the calculated results of the mechanical energy and the electromechanical efficiency, and Table 5 summarizes their value with measured actuation stroke. 

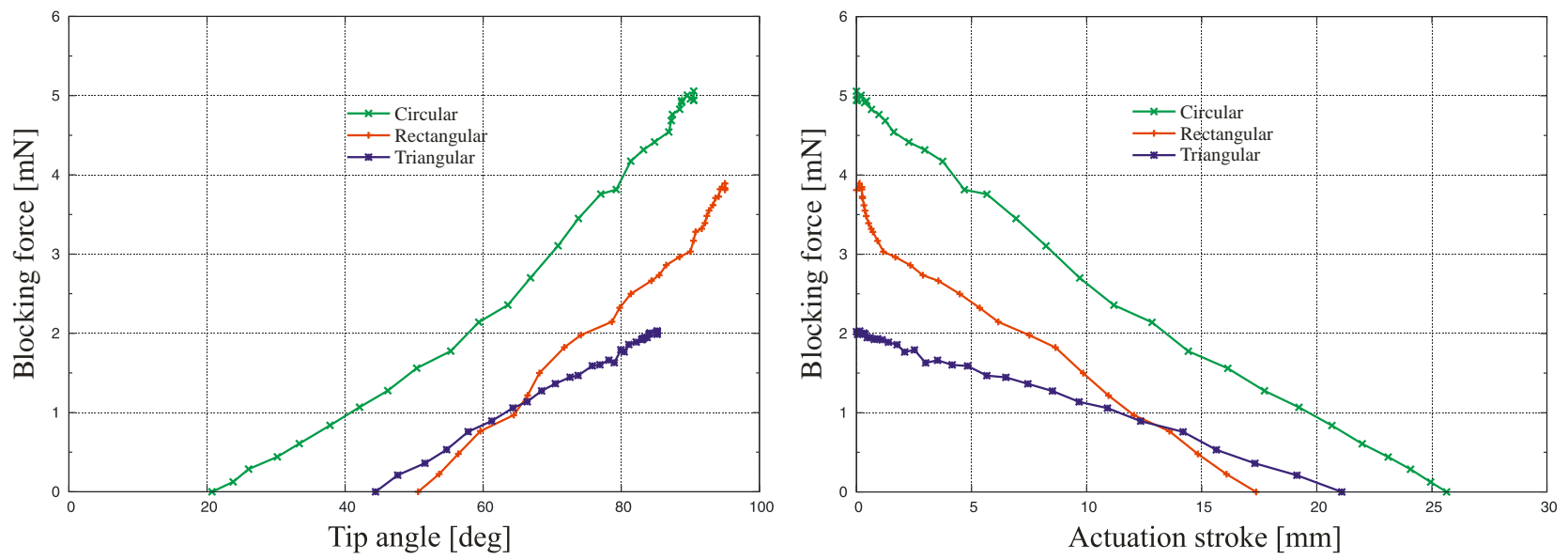

Figure 12. (Left) measured tip angle as a function of applied voltage, and (right) measured actuation stroke versus blocking force. Circular shape shows higher value on both the actuation stroke and the blocking force. Rectangular shape shows the maximum force higher than triangular shape, while the stroke is smaller.

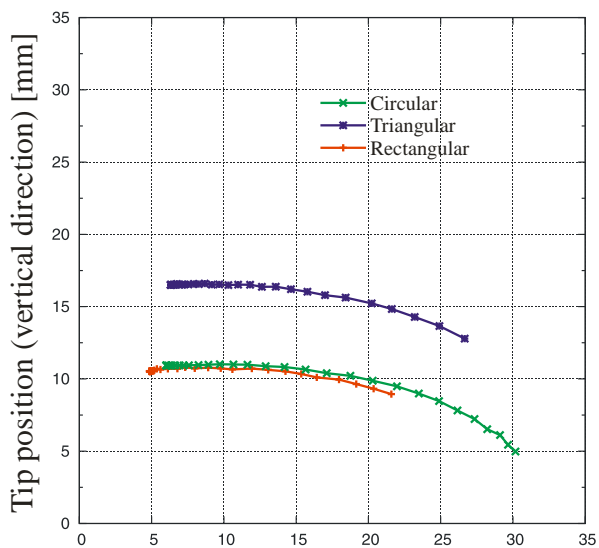

Tip position (length direction) $[\mathrm{mm}]$

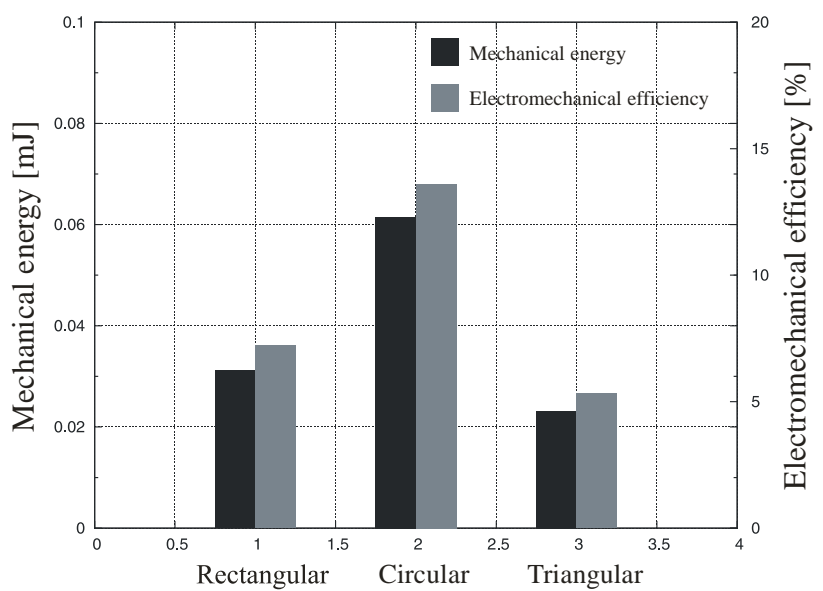

Rectangular Circular Triangular

Figure 13. (Left) measured tip position in the length direction and in the vertical direction, and (right) calculated mechanical energy and electromechanical efficiency. Triangular shape shows higher position in the vertical direction, as it has longest frame length. Circular shape shows longest actuation stroke, and exhibited mechanical energy three times as high as triangular shape.

Table 5. Summary of measured actuation stroke and calculated performance of the actuators.

\begin{tabular}{|c|c|c|c|}
\hline Frame shape & Rectangular & Circular & Triangular \\
\hline Actuation stroke angle (deg) & 44.4 & 69.8 & 40.8 \\
\hline Actuation stroke (mm) & 17.4 & 25.6 & 21.1 \\
\hline Mechanical energy (mJ) & 3.1 & 6.2 & 2.3 \\
\hline Electromechanical efficiency (\%) & 7.2 & 13.6 & 5.3 \\
\hline
\end{tabular}




\section{DISCUSSION}

In the experiment on different frame width described in subsection 4.2, tip angles at initial state $(\mathrm{V}=0)$ are shifted by around $30 \mathrm{deg}$, as shown in Figure 6 (left). This is due to variation of the bending stiffness corresponding to frame width. The bending stiffness of a beam can be written as $E I$ where $E$ is the elastic modulus, and $I$ is the second moment area of the beam cross section. If the beam has rectangular cross-section, the second moment area $I$ can be described as:

$$
I=\frac{b h^{3}}{12}
$$

where $b$ is the width, and $h$ is the height of the cross-section. In our case, $b$ is simply increased. The effect of the bending stiffness change appears in Figure 6 as: the wider the frame, the smaller the stroke, and the wider the frame, the larger the force. It can be considered that the relation, that is, the trade-off between the stroke and the blocking force is exposed here, and it shows design capability of actuators as adjusting bending stiffness.

In the experiment on different pre-stretch ratios reported in subsection 4.3 , there is a difference of the initial tip angle among the pre-stretch ratios as shown in Figure 7 (left). We assume this difference is caused by strength of the prestretch on $Y$ direction, and hence the combination 1.3:1.95 has largest angle. The initial angle of the combination 1.95:1.3 is larger than 1.3:1.3, which is due to the contribution of additional pre-stretch caused by the pre-stretch on $X$ direction. For example, if a membrane is stretched in the width direction, the membrane tends to contract in the length direction. As shown in Table 3, actuation stroke is larger at 1.95:1.3. It can be considered that strong pre-stretch in $X$ direction contributes to actuation strain in $Y$ direction. For example, when a DEA is pre-stretched along the width direction, expansion of the DEA is greater along the length direction because Young's modulus is higher in that direction. This phenomenon has been reported in [20]. The combination 1.3:1.95 has actuation stroke larger than 1.3:1.3. This is a contribution of strong pre-stretch on $Y$ direction to $X$ direction same reason as mentioned above. Since all the actuators have same frame shape, it can be assumed that the magnitude of the blocking force simply refers that of the actuation stroke, as shown in Figure 7 (right). From the result, on the actuators, we assume that there is an optimal point of the prestretch ratio with the combination of higher stretch in the direction $X$ and lower stretch in the direction $Y$, where optimized pre-stretch for the maximal actuation performance with required deformation shape (initial angle) is realized.

In the experiment on different frame shape explained in subsection 4.4, circular shape shows largest actuation stroke, as shown in Figure 9 (left). We assume that the shape of the electrodes contributed to the stroke difference, that is, circular shape thus symmetric DEA expands easily compared to other shapes. In the rectangular DEA boundary, actuation strain of the DEA is restricted along the edge where the membrane is fixed. In the circular boundary, the actuation strain is perpendicular to the edge at any point, thus the membrane has less constraint. Triangular shape has smallest actuation stroke because of unsymmetric of the boundary on the width direction. Also, there is effect of initial shape of the frame. The deformation of the triangular frame includes not only in $Y$ (length) direction but also in $X$ (width) direction stronger than other frame. Even the deformation results in bending along the length direction, it produces less actuation for $Y$ direction because more actuation is needed for $X$. In Figure 12 (right), the blocking force of circular shape is larger than the others at every stroke position, which results in highest value of the mechanical energy and its efficiency. This simply means that either the frame shape or the DEA shape is effective to the actuation performance. Therefore, the actuation performance can be optimized in terms of the frame shape, the DEA shape, or their combination.

\section{CONCLUSION}

In this study, we characterized the actuation behavior of cm-scale DEMES in order to understand the effect of the mechanical parameters and their relation, and thus to be able to optimize the design, with the goal of designing a soft robot based on interconnected self-sensing DEMES. Two key novelties were the use of flex-PCB as the frame (thus allowing electrical signal routing in addition to mechanical function) and the use of silicone membranes (no visco-elastic response, can cast any desired thickness, and providing one more free parameter). The results showed a clear path to actuator optimization for a given task, and the trade-offs that are involved.

Our future work includes further investigation of the relation between the frame shape and the DEA shape on the actuator performance, and the inclusion of self-sensing/switching architecture for a modular soft robot. 


\section{ACKNOWLEDGMENTS}

We gratefully acknowledge financial supports from the Swiss National Center for Competence in Research (NCCR) Robotics (Project No. 1.2 Sensorymotor tissues), and from the Swiss National Science Foundation, grant \#200020140394

\section{REFERENCES}

[1] D. Trivedi, C. D. Rahn, W. M. Kier, and I. D. Walker, "Soft robotics: Biological inspiration, state of the art, and future research," Applied Bionics and Biomechanics 5(3), pp. 99-117, 2008.

[2] F. Iida and C. Laschi, "Soft Robotics: Challenges and Perspectives," Procedia Computer Science 7, pp. 99-102, 2011.

[3] R. Pfeifer, M. Lungarella, and F. Iida, "The Challenges Ahead for Bio-Inspired 'Soft' Robotics," Communications of the ACM 55(11), pp. 6-87, 2012.

[4] R. E. Pelrine, R. D. Kornbluh, and J. P. Joseph, "Electrostriction of polymer dielectrics with compliant electrodes as a means of actuation," Sensors and Actuators A: Physical 64(1), pp. 77-85, 1998.

[5] B. Paul, and Q. Pei. "Advances in Dielectric Elastomers for Actuators and Artificial Muscles," Macromolecular Rapid Communications 31(1), pp. 10-36, 2010.

[6] S. Rosset, and H. R. Shea. "Flexible and Stretchable Electrodes for Dielectric Elastomer Actuators," Applied Physics A: Materials Science 110(2), pp. 281-307, 2012.

[7] F. Carpi, D. D. Rossi, R. Kornbluh, R. Pelrine, and P. Sommer-Larsen, "Dielectric Elastomers as Electromechanical Transducers," Elsevier, pp. 14, 2008.

[8] I. W. Hunter, J. D. Madden, N. Vandesteeg, P. G. Madden, and A. Takshi, "Artificial Muscle Technology: Physical Principles and Naval Prospects," Oceanic Engineering 29(3), pp. 706-728, 2004.

[9] S. Michel, X. Q. Zhang, M. Wissler, C. Löwe, and G. Kovacs, "A comparison between silicone and acrylic elastomers as dielectric materials in electroactive polymer actuators," Polymer International 59(3), pp. 391-399, 2010.

[10] L. A. Toth and A. A. Goldenberg, "Control System Design for a Dielectric Elastomer Actuator: the Sensory Subsystem," in Proceedings of SPIE - The International Society for Optical Engineering, 4695, pp. 323-334, 2002.

[11] R. Pelrine, R. Kornbluh, J. Eckerle, P. Jeuck, S. Oh, Q. Pei, and S. Stanford, "Dielectric Elastomers: Generator Mode Fundamentals and Applications," in Proceedings of SPIE - The International Society for Optical Engineering, 4329, pp. 148-156, 2001.

[12] B. M. O’Brien, E. P. Calius, T. Inamura, S. Q. Xie, and I. A. Anderson, “Dielectric elastomer switches for smart artificial muscles," Applied Physics A: Materials Science \& Processing 100(2), pp. 385-389, 2010.

[13] A. O'Halloran, F. O'Malley, and P. McHugh "A review on dielectric elastomer actuators, technology, applications, and challenges," Applied Physics 104(7), p. 071101, 2008.

[14]I. A. Anderson, T. A. Gisby, T. G. McKay, B. M. O’Brien, and E. P. Calius, "Multi-functional Dielectric Elastomer Artificial Muscles for Soft and Smart Machines," Applied Physics 112(4), p. 041101, 2012.

[15] G. Kofod, M. Paajanen, and S. Bauer, "Self-organized minimum-energy structures for dielectric elastomer actuators," Applied Physics A: Materials Science \& Processing 85(2), pp. 141-143, 2006.

[16] G. Kofod, W. Wirges, M. Paajanen, and S. Bauer, "Energy minimization for self-organized structure formation and actuation," Applied Physics Letters 90(8), pp. 081916, 2007.

[17] X. Zhao and Z. Suo, "Method to analyze programmable deformation of dielectric elastomer layers," Applied Physics Letters 93(25), p. 251902, 2008.

[18]B. O’Brien, T. McKay, E. Calius, S. Xie, and I. Anderson, "Finite element modelling of dielectric elastomer minimum energy structures," Applied Physics A: Materials Science \& Processing 94(3), pp. 507-514, 2009.

[19]C. Keplinger, M. Kaltenbrunner, N. Arnold, and S. Bauer, "Röntgen's electrode-free elastomer actuators without electromechanical pull-in instability," Proceedings of the National Academy of Sciences 107(10), pp. 4505-4510, 2010.

[20] M. T. Petralia and R. J. Wood, "Fabrication and analysis of dielectric-elastomer minimum-energy structures for highly-deformable soft robotic systems," in Proceedings of IEEE/RSJ International Conference on Intelligent Robots and Systems, pp. 2357-2363, 2010. 
[21]P. J. White, S. Latscha, S. Schlaefer, and M. Yim, "Dielectric Elastomer Bender Actuator Applied to Modular Robotics," in Proceedings of IEEE/RSJ International Conference on Intelligent Robots and Systems, pp. 408413, 2011.

[22] "DuPont ${ }^{\mathrm{TM}}$ Pyralux ${ }^{\circledR}$ AP Technical Data Sheet," http://www2.dupont.com/Pyralux/en_US/assets/downloads/pdf/APclad_H-73241.pdf

[23] S. Rosset, M. Niklaus, P. Dubois, Philippe, M. Dadras, and H. R. Shea, "Mechanical properties of electroactive polymer microactuators with ion-implanted electrodes," in Proceedings of SPIE - The International Society for Optical Engineering, 6524, p. 652410, 2007.

[24] S. Rosset, M. Niklaus, P. Dubois S. Rosset, M. Niklaus, P. Dubois, and H. R. Shea, "Metal Ion Implantation for the Fabrication of Stretchable Electrodes on Elastomers," Microelectromechanical Systems 19, pp. 470-478, 2009. 